\title{
A Revised Deep Belief Network for Predicting the Slurry Concentration of a Cutter Suction Dredger
}

\author{
Changyun Wei ${ }^{a}$, Fusheng $\mathrm{Ni}^{\mathrm{b}}$ and Jinbao Yang ${ }^{\mathrm{c}}$ \\ College of Mechanical Engineering, Hohai University, Changzhou 213022, China; \\ ac.wei@hhu.edu.cn, bfushengni@163.com, cjin_baoyang@163.com
}

Keywords: Cutter Suction Dredger, Slurry Concentration, Deep Belief Network, Classifier.

\begin{abstract}
In order to predict the slurry concentration of a Cutter Suction Dredger (CSD), a revised Deep Belief Network (DBN) that contains two classifier models is proposed in this work. The two classifier models (i.e., a constant step model and a probability sampling model) are used to process the original data captured in a CSD during a dredging project. Then the classifier models are employed to build the revised DBN to predict the slurry concentration of a CSD. The simulated results show that the proposed approach can effectively extract the features of working data, and also predict the slurry concentration efficiently.
\end{abstract}

\section{Introduction}

The cutter suction dredger (CSD) is one of the most widely used equipment in dredging engineering, and the slurry concentration is an important indicator of its production [1]. In dredging projects, the excavation and transportation process of underwater soil is complex and dynamic, and there are many interrelated factors that affect the slurry concentration. Thus, even if the vacuum degree, traverse speed and other process parameters are known, we cannot timely and accurately predict the trend of slurry concentration, which can result in low efficiency of dredging operations, as well as the dangers of pipeline clogging. Therefore, it is necessary to study the variation of the CSD slurry concentration with the influence factors in order to provide a basis for improving the production efficiency and achieving the optimal control. At present, the work [2] [3] build prediction models that still lack of accuracy. Moreover, those models cannot extract the relevant rules of construction data. However, the operators may not be very concerned on the specific value of data, and often only need to know that the data remain in a certain range. Therefore, this paper adopts the idea of classification, and two classifier models for the Deep Belief Network are proposed.

\section{Revised Deep Belief Network}

In this paper, the Deep Belief Network (DBN) is used to build the concentration prediction model, and the principles and methods of a DBN are presented in [4 8]. The DBN can automatically extract the deep features contained in the data, but the features extracted are still a series of isolated features, and cannot get a series of rules of the input data. Therefore, a feature classifier is designed to obtain a set of rules for input data. Then, these rules are input into the DBN, and the output of the network is evaluated to judge the quality of the classifier in order to improve the feature classifier. The input factors of the prediction model are the vacuum degree, the current of a cutter head, the swing speed, the velocity of flow, and the output factor is the slurry concentration.

\subsection{Classifier Models}

In this paper, two classifiers model are proposed, which are constant step and probability sampling, and a concentration prediction model of the CSD based on classified data is established. Two classifier models of constant step and probability sampling are used to classify four input factors (i.e., the vacuum degree, the current of a cutter, the swing speed, the velocity of the hydraulic pipeline transportation) and an output factor which is the slurry concentration. In this way, two different classifications of each factor are obtained. Then the DBN algorithm is used to predict and analyze the advantages and disadvantages of the two classifications, and the final classification strategy of the 
input and output factors is determined. Finally, the prediction model of slurry concentration is constructed by using the classified data, and the prediction performance of the model is compared and analyzed. Particularly, when the specific classification of the factors was determined, the other variables were analyzed using the original data.

- Constant Step Classifier: Constant step classifier is actually an averaging classification method. For a set of data, we can get the maximum / minimum value, which can determine the total length of the data set. When the number of groups is given, the group spacing of each group can be determined according to Formula 1, and then the group processing is carried out: $\Delta L=\left(L_{1}-L_{2}\right) / L_{0}$

Where $L_{1}$ represents the maximum value of the set of data, $L_{2}$ represents the minimum, $L_{0}$ represents the number of the groups, and $\Delta L$ represents the group spacing.

- Probability Sampling Classifier: Probability sampling classifier is actually based on probability distribution for data classification. It still determines the group spacing for each set according to Formula 1. Only at this point, $L_{1}$ and $L_{2}$ represent the maximum and minimum values of the interval $L_{1}$ and $L_{2} . L_{0}$ indicates the number of groups of the next round in the interval, which can be determined by the Formula 2:

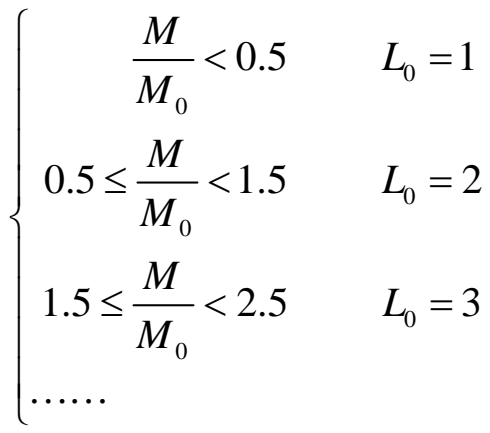

Where $M$ represents the amount of data between the interval $L_{1}$ and $L_{2} . M_{0}$ Represents the amount of data for each class that is divided into N0 classes, which can be obtained by:

$M_{0}=N / N_{0}$

Where $\mathrm{N}$ represents the total number of categorical data.

\section{Model Design and Analysis}

\subsection{The Swing Speed}

Taking the traverse speed as an example, the detailed classification process of the two classification methods is described in detail. Firstly, the probability sampling classification method is adopted, and the classification process is as follows:

- Obtain the maximum and minimum values of the swing speed and the total amount of data (i.e., $\mathrm{N})$. It is shown in step one of Fig. 1.

- The data of the swing speed is averagely divided into two groups, and compute each set of the data as N1 and N2. Then compute the amount of data in each group in theory as N0 when it is averagely divided into two groups, as shown in Fig. 1, step two.

- Compare the size of N1, N2 and N0, and determine the next group number according to the sampling algorithm is given by Formula 2. Then again calculate each group spacing and each group of data, and the theoretical calculation of each set of data.

- Repeat the above steps, and the specific classification is shown in Fig. 1. 


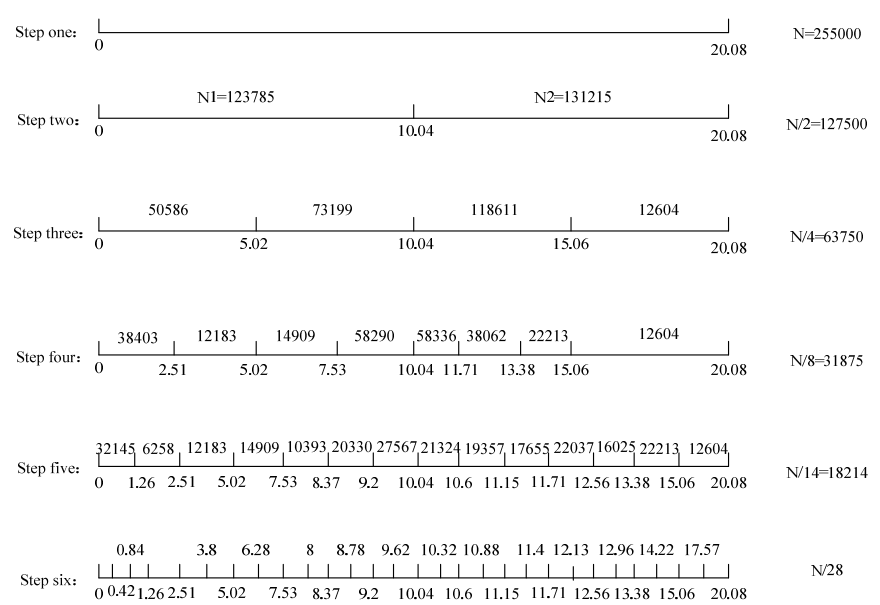

Fig. 1 the sketch map of the classification of the swing speed.

From the step six of Fig. 1, we can see that the interval has the focus swing speed is relatively small when the group iterates to this step. Then, we will not only increase the group number but also increase the packet error, and it is not conducive to the construction of rule extraction traverse speed. If we continue to divide it, it will not only increase the number of groups, but also increase the group error, and it is also not conducive to extracting the construction rules of traverse speed. Thus, when this step is stopped, the velocity of the traverse is divided into 28 groups. So the method of constant step classification is divided into 28 groups as well. As we know, the swing speed is between [0, 20.08]. In order to facilitate the calculation, the interval is changed to [0,21]. The distance between 0.75 groups is determined by Formula 1 through the constant step classification method, and the classification results are shown in Fig.2.

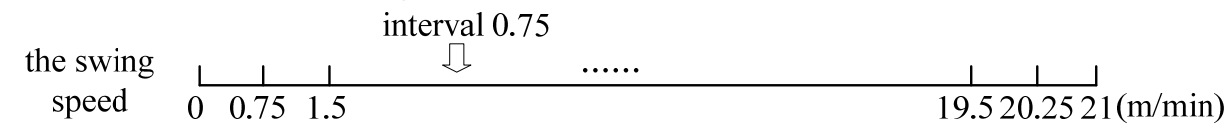

Fig. 2 Classification results of the swing speed.

We can respectively change the raw data of the swing speed to the classification data obtained by the two classification methods, and other factors are kept constant in the concentration prediction model, which is trained by DBN algorithm. The prediction results are shown in Fig.3. As shown in Fig. 3, for the traverse speed, the prediction results obtained by the probability sampling classifier are slightly better than the results obtained by the constant step classifier, which can also be obtained by Table 1. Therefore, the classification method of the swing speed is selected as the probability sampling method.

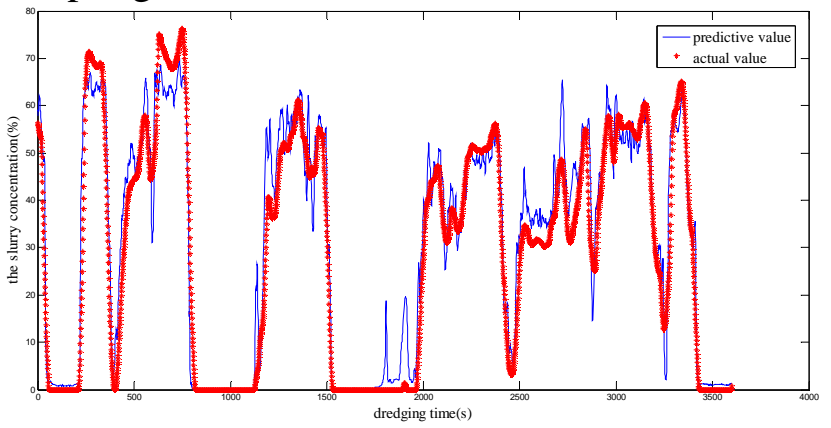

a) probability sampling

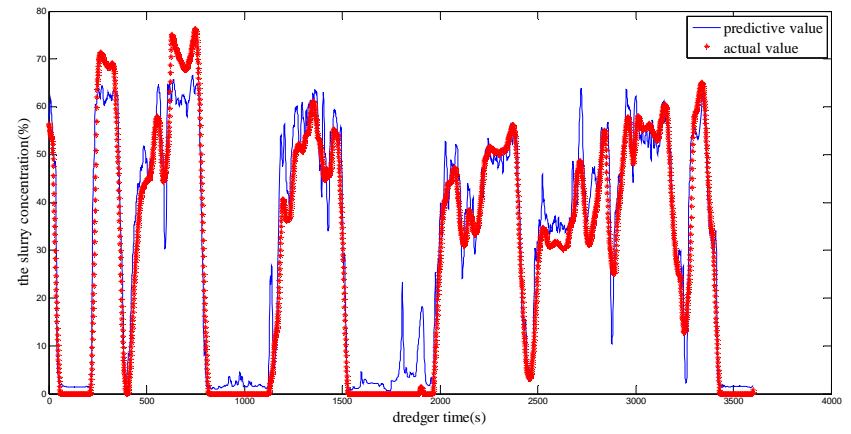

b) constant step

Fig. 3 the prediction results of two classifiers of the swing speed.

Table 1. Comparison of prediction results of different classification of the swing speed.

\begin{tabular}{ccc}
\hline classifier & MAE & MSE \\
\hline probability sampling & 4.2521 & 40.0867 \\
constant step & 4.4047 & 41.6629 \\
\hline
\end{tabular}




\subsection{The Vacuum Degree}

The vacuum degree is classified by the same classification method, and the specific classification step and the traverse speed classification are the same. The final classification result is shown in Fig. 4, in which the vacuum degree is divided into 35 categories. The vacuum data is classified and trained by the DBN algorithm, and the results are shown in Fig. 5.

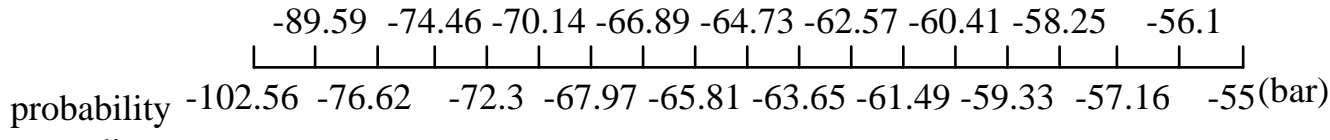

$$
\begin{aligned}
& \text { sampling }
\end{aligned}
$$

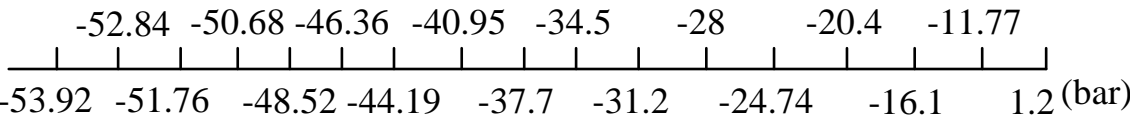

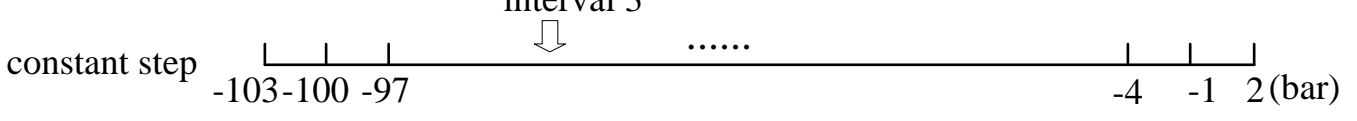

Fig. 4 Classification results of the vacuum degree.

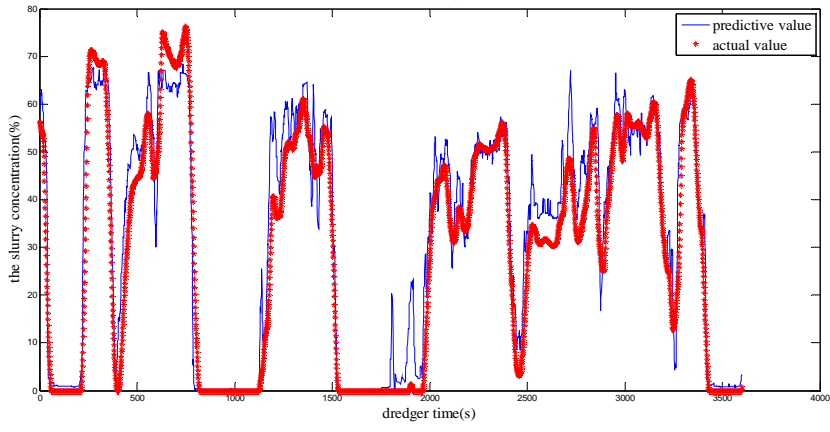

a) probability sampling

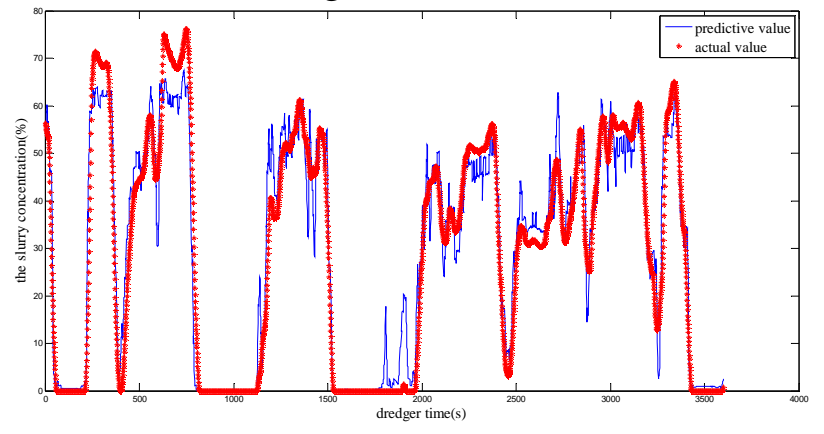

b) constant step

Fig. 5 The prediction results of two classifiers of the vacuum degree.

As can be seen from Fig.5, the classification data obtained by the two classifiers both can better predict the slurry concentration for the vacuum degree. The performance parameters of the DBN prediction model obtained by the two classifiers are shown in Table 2. The MAE and MSE of the prediction model obtained by the constant step classification method are slightly smaller than the probability sampling classification method from Table 2. Therefore, a constant step classification method is selected for the vacuum degree.

Table 2. Comparison of prediction results of different classification of the vacuum degree.

\begin{tabular}{ccc}
\hline classifier & MAE & MSE \\
\hline probability sampling & 4.9340 & 52.4882 \\
constant step & 4.3700 & 40.1455 \\
\hline
\end{tabular}

\subsection{The Current of the Cutter Head}

The current of a cutter also uses the same classification method, and the classification results obtained as shown in Fig. 6. It is divided into 22 categories.

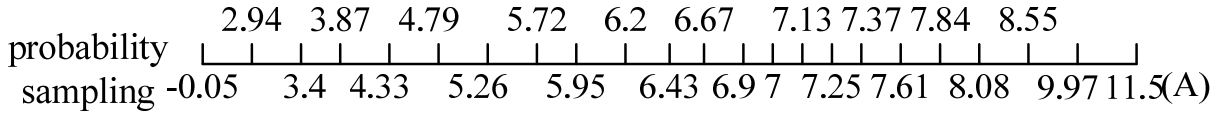

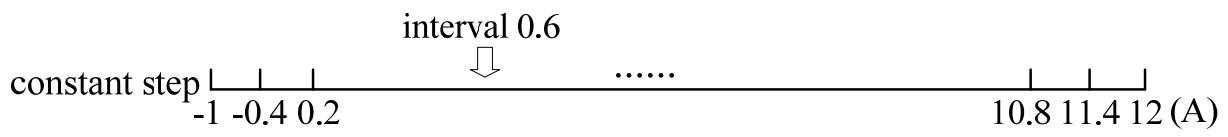

Fig. 6 Classification results of the current of a cutter.

The prediction results of the concentration prediction model are shown in Fig. 7. The related performance parameters of the prediction model are shown in Table 3 for the current of a cutter. From Fig. 7 and Table 3 we can see that the performance of concentration prediction model which is built 
by the data of the current of a cutter obtained by constant step classification method is better. Therefore, a constant step classification method is selected for the current of a cutter.

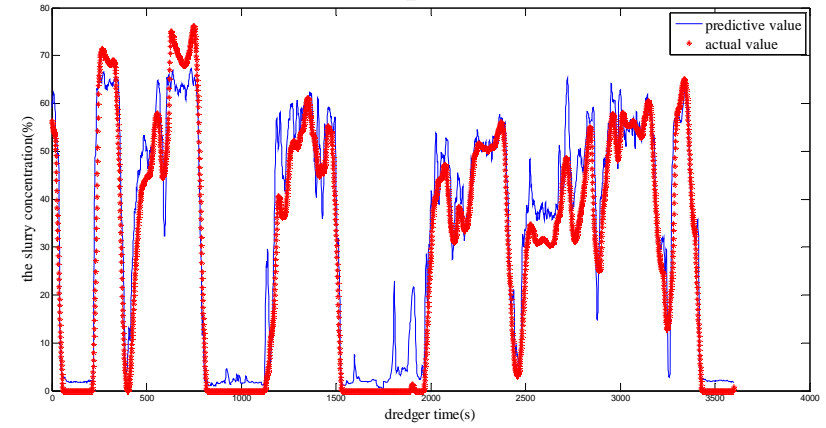

a) probability sampling

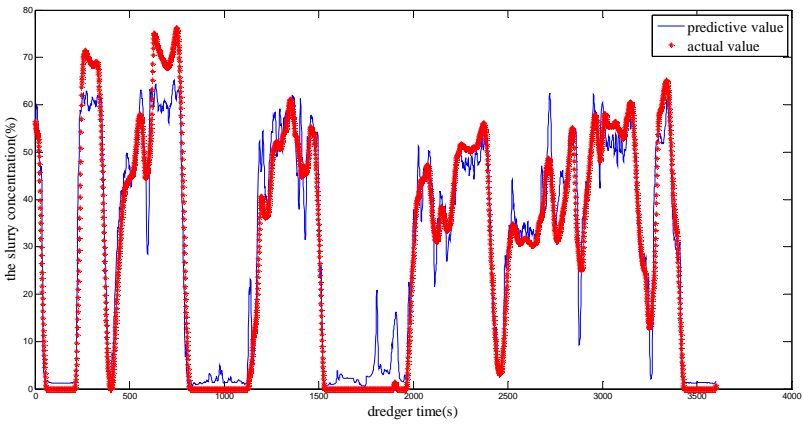

b) constant step

Fig. 7 the prediction results of two classifiers of the current of a cutter

Table 3. Comparison of prediction results of different classification of the current of a cutter.

\begin{tabular}{ccc}
\hline classifier & MAE & MSE \\
\hline probability sampling & 5.2313 & 50.3516 \\
constant step & 4.3782 & 35.6560 \\
\hline
\end{tabular}

\subsection{The Velocity of Flow}

The same classification method is used for the velocity of flow, and the final result is shown in Fig. 8. The velocity of flow are divided into 14 categories. The prediction results of the concentration prediction model are shown in Fig. 9. For the velocity of flow, the performance parameters of the concentration prediction model obtained by the two classifiers are shown in Table 4.

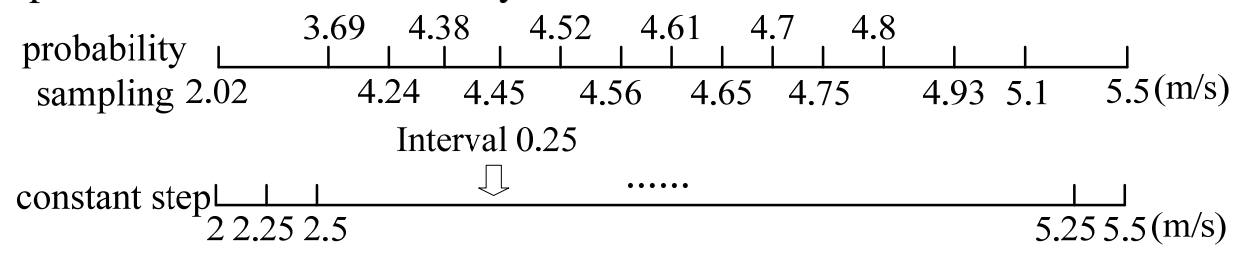

Fig. 8 Classification results of the velocity of flow.

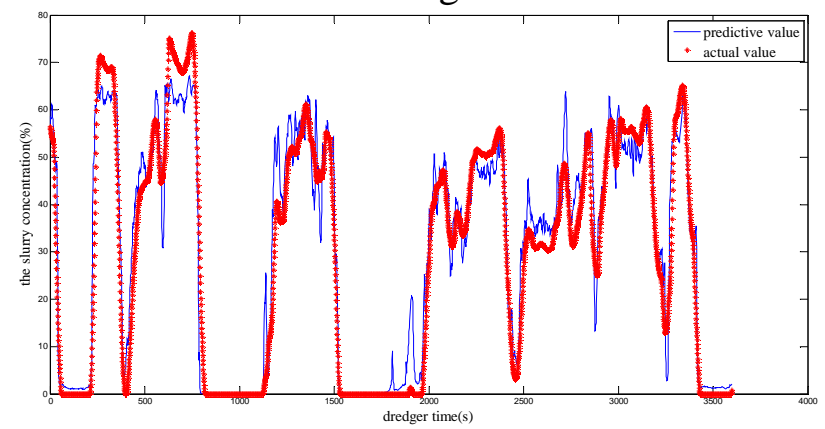

a) probability sampling

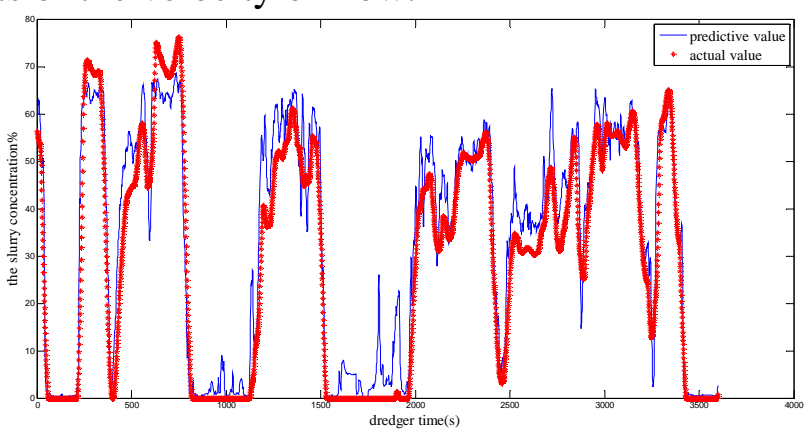

b) constant step

Fig. 9 the prediction results of two classifiers of the velocity of flow

Table 4. Comparison of prediction results of different classification of the velocity of flow.

\begin{tabular}{ccc}
\hline classifier & MAE & MSE \\
\hline probability sampling & 4.3470 & 39.8035 \\
constant step & 5.3363 & 54.4486 \\
\hline
\end{tabular}

Fig. 9 and Table 4 shows that the prediction performance of the concentration prediction model which is established by the method of probability sampling is better, and the prediction curve and actual curve is more consistent. At the same time, the corresponding performance parameter of MAE and MSE was significantly less than the constant step classification method. Therefore, a probability sampling classification method is selected for the velocity of flow. 


\subsection{The Slurry Concentration}

The classification results of the slurry concentration are shown in Fig.10. It is divided into 26 categories. The prediction results of the concentration prediction model are shown in Fig. 11. For slurry concentration data, the performance parameters of the concentration prediction model obtained by the two classifiers are shown in Table 5.

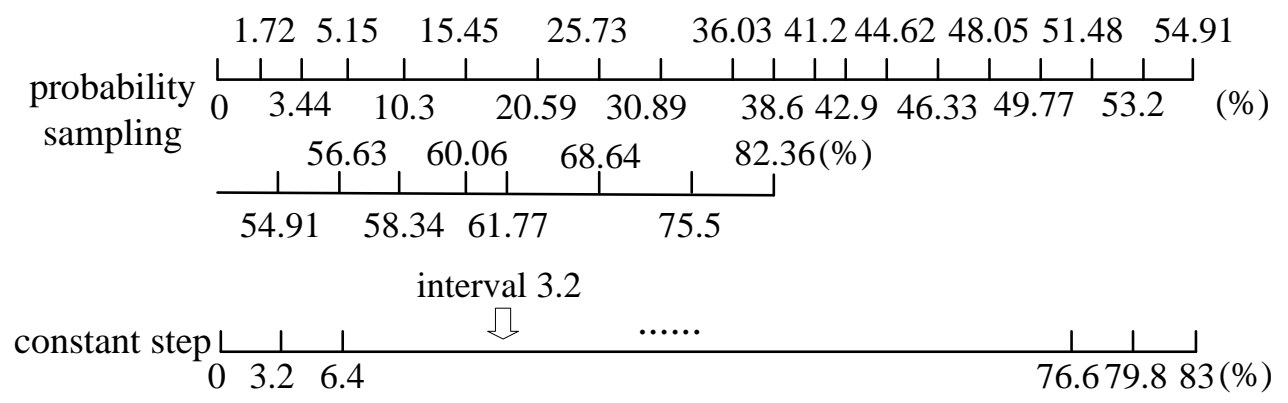

Fig. 10 Classification results of the slurry concentration.

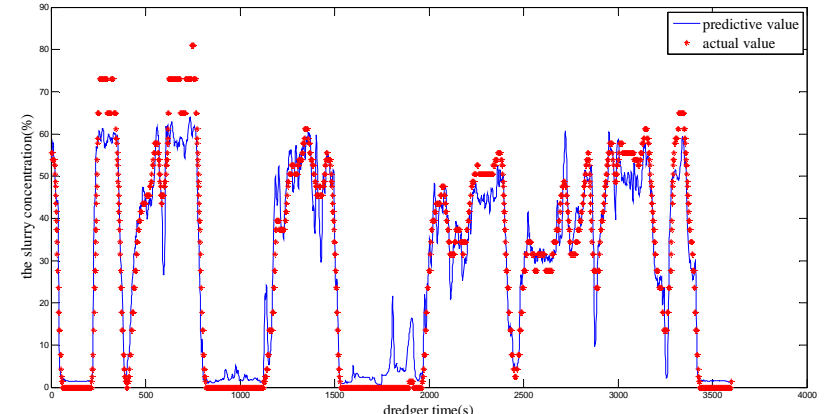

a) probability sampling

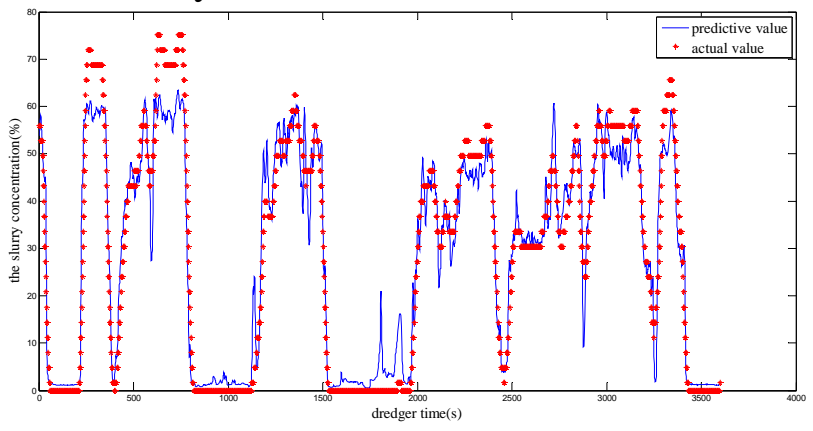

b) constant step

Fig. 11 The prediction results of two classifiers of the slurry concentration.

Table 5. Comparison of prediction results of different classification of the slurry concentration.

\begin{tabular}{ccc}
\hline classifier & MAE & MSE \\
\hline probability sampling & 4.7191 & 40.4611 \\
constant step & 4.4391 & 37.1172 \\
\hline
\end{tabular}

Fig. and Table 5 shows that the prediction performance of the concentration prediction model which is established by the method of constant step is better. Therefore, a constant step classification method is selected for the slurry concentration.

\section{Results}

Through the above analysis, the classification strategy of input and output factors has been selected. The swing speed and the velocity of flow are classified by probability sampling. The vacuum degree and the current of a cutter and the slurry concentration are classified by constant step. A concentration prediction model is constructed based on these selected classification strategies, and the predicted results are shown in Fig. 12.

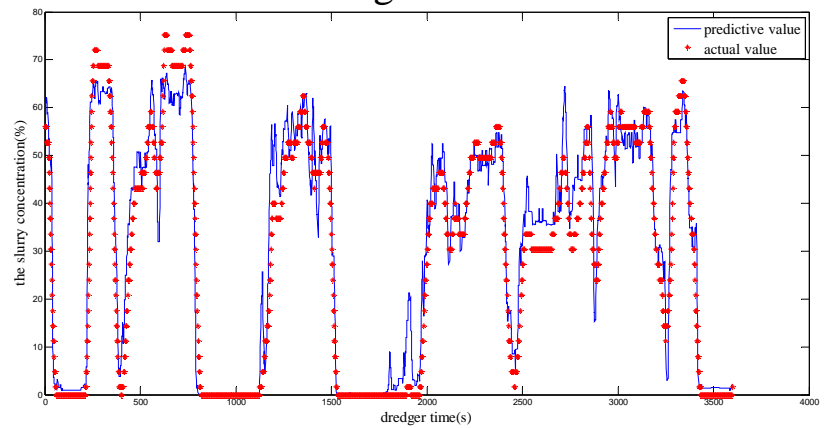

a) categorical data

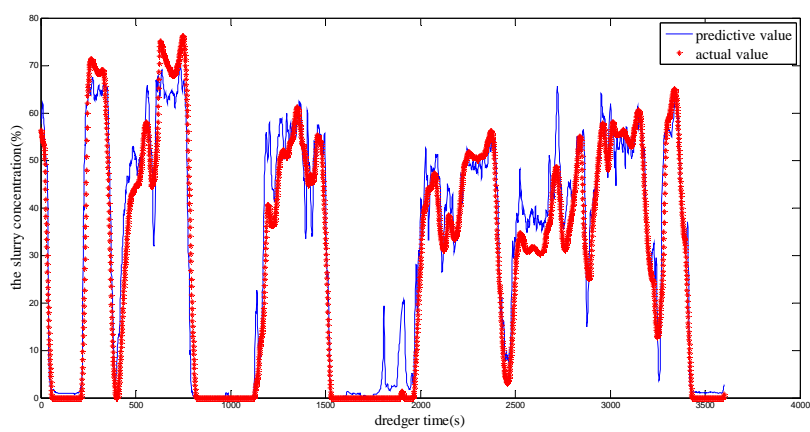

b) primary data

Fig. 12 The prediction curve of the slurry concentration. 
As shown in Fig. 12a) and 12b), the prediction model established after the classification of the original data is better than the concentration prediction model established in the absence of classification. As shown in Table 6, the MAE and MSE values of the predicted model after classification are less than the values corresponding to the raw data.

Table 6. Comparison of different concentration prediction classifiers.

\begin{tabular}{ccc}
\hline classifier & MAE & MSE \\
\hline probability sampling & 4.2445 & 37.6589 \\
constant step & 4.6357 & 40.3741 \\
\hline
\end{tabular}

Based on the above analysis, the optimized concentration prediction model not only achieves better prediction of the slurry concentration but also can extract a series of rules of input and output data. These rules have certain guiding significance to the construction process of the cutter suction dredger. The swing speed is divided into 28 categories, and the vacuum degree is divided into 35 categories. The current of a cutter is divided into 22 categories, the velocity of flow is divided into 14 categories, and the slurry concentration is divided into 26 categories. Thus, a rule table for dredging and conveying process of the cutter suction dredger is formed. The table contains at most 7.84 million rules, and these rules must be far less than the rules of the raw data. These rules can be used to guide the construction of the CSD, and can also be applied to the expert system of cutter suction dredger.

\section{Conclusion}

Two classifier design methods are proposed in this paper. The original data are classified, and the concentration prediction model of the CSD based on classified data is established. The prediction results show that the model has better prediction performance, and can effectively predict the slurry concentration for a cutter suction dredger. Moreover, it also can extract relevant features of construction data, which has certain directive significance to establish the knowledge base of an expert system for cutter suction dredgers.

\section{Acknowledgments}

This research was supported by the Fundamental Research Funds for the Central Universities (2015B30114).

\section{References}

[1]. Fusheng Ni. Summary of development of dredging equipment at home and abroad. Journal of Hohai University(Changzhou Campus), 2004, 18 (1): 1-9.

[2]. Huanmin $\mathrm{Xu}$, Feng Zhou. Study on mathematical model of dredging technology based on regression analysis. Mechanical design and manufacturing engineering, 2016, 45 (8): 78-81.

[3]. Zhiqiang Li. Dredging optimization of cutter suction dredger. Jiangsu University of Science and Technology, 2011.

[4]. Bengio Y. Learning deep architectures for AI. Foundations and trends ${ }^{\circledR}$ in Machine Learning, 2009, 2(1): 1-127.

[5]. Hinton G E, Osindero S, Teh Y W. A fast learning algorithm for deep belief nets. Neural computation, 2006, 18(7): 1527-1554.

[6]. Fischer A, Igel C. An introduction to restricted Boltzmann machine. Iberoamerican Congress on Pattern Recognition. Springer Berlin Heidelberg, 2012: 14-36.

[7]. Hinton G. A practical guide to training restricted Boltzmann machines. Momentum, 2010, 9(1): 926.

[8]. Juan Tan, Shengchun Wang. Study on traffic congestion prediction model based on deep learning. Computer application research, 2015, 32 (10): 2951-2954. 